\title{
Gender Bias on Tinder: Transforming an Exploratory Qualitative Survey into Statistical Data for Contextualized Interpretation
}

\author{
Milena Ribeiro Lopes and Carl Vogel \\ Computational Linguistics Group, Centre for Computing and Language Studies, \\ School of Computer Science and Statistics, \\ Trinity College Dublin, the University of Dublin, Ireland \\ lopsem@tcd.ie vogel@tcd.ie
}

\begin{abstract}
Tinder is an online dating application that enables a humanhuman interaction. However, the ease of connection brings to light some concerns about possible harmful gender dynamics, which can enhance bias within technological developments and threaten women empowerment. This research aims to investigate whether the application meets women's expectations, whether there is gender bias and sexist behavior during the users' experiences and what is the relation between the findings to the graphical user interface. The research approaches to a mixed method design in order to achieve its goals.
\end{abstract}

Keywords: online dating applications, gender bias, mobile interfaces

\section{Introduction}

Interface design is an area of inquiry deeply influenced by aesthetic response. The aesthetic of interfaces to interactive systems is expressed partly in the graphical user interface (GUI), which guides users through human-device dialogue. Interactive systems offer users an opportunity to users to interact with the each other and the world through complex symbolic representations [1].

Aesthetic judgement is quintessentially qualitative. However, one may meaningfully and productively also address quantitative questions in relation to aesthetic judgements, perhaps most obviously exploring the extent to which such judgements are shared and demographic or environmental variables that correlate with judgements of particular categories. One may also look to the internal properties of such judgements and their relationships to other judgements. In pursuing such analysis of qualitative questions, it is useful to develop a method that supports the integration of quantitative information. This does not reduce qualitative judgement to quantitative response, but informs the context of interpretation for the qualitative judgement. Indeed, this is the methodological integration of quantitative methods into qualitative research for which we argue: quantitative method informs the context of interpretation for qualitative methods. Equally, it is important to emphasize: the integrative method is not 
reductionist of qualities to quantities. Emphasis on the context of interpretation is perhaps an aspect of this methodological approach that sets it apart from other qualitative approaches informed by quantitative analysis. To clarify our discussion further, we ground the ideas in a case study of design for human-human computer mediated communication interfaces.

Online dating application use is a current trend among young people, and its success can be attributed to the ubiquity of mobile technology, to the ease and privacy of virtuality and to the eternal importance of human relationships. Tinder is a popular example. However, the concept of relationships mediated by mobile applications leads to reflection on gender differences and the concern about possible sexist behaviors. We discuss findings regarding sexist behavior evidently established through the Tinder GUI and which have design implications that, if acted upon, could improve the experience of women users of Tinder.

The idea of this study emerged from informal observations and conversations with female users about Tinder in general, not directed to feminist discussion. It was clear that they experienced contradictory feelings regarding the application - they indicated that they would encourage others to use it although they had frustrating experiences; they would install and uninstall it multiple times; they would express directly opposing responses: some moments of total excitement (beautiful people, new matches, nice dialogues) and others of massive complaint (ugly people, no interaction, time-wasting, disrespectful approaches). From this observation of conflicting responses emerged the hypothesis that Tinder might not consider female needs and might be a space for the reinforcement of sexist behavior that is expressed through the interaction logic and its GUI.

We defined three research objectives that would address this question. The first objective of the study reported here was to learn how women perceive their experience on Tinder. The second objective was to investigate whether there are sexist patterns of behavior during the Tinder experience. The third objective was to check which elements of the interface contribute to a good or bad experience regarding gender dynamics. Some findings show positive aspects of the application and other findings show its negative sides. The idea was to provide foundations for a holistic view of perceptions of the system.

The first objective would guide the research to understand how women feel during their experience on Tinder, in general, so we could evaluate if there is a problem to solve through design or not (with consequences to the continuation with further studies), the second would point out whether there are sexist behavior that diminishes the overall perception of the experience and the third objective would enable to understand which aspects of the interface design contributes to the establishment of a imbalanced gender dynamic.

\section{User experience and the graphical user interface}

Interaction with mobile devices can be understood as a form of multimodal communication in which users and devices interact with each other through different dimensions. These dimensions of interaction happen through different commu- 
nication channels such as speech, audio, touch, gesture, and graphics. Mobile devices can process sounds, gestures, gaze movement and touch. They can return other sounds (audio), vibration (haptics) and visual information (graphics). This computer feedback is based on human visual, auditory and tactile sensory capacities [2]. This relation between inputs and outputs characterize the multimodal interaction. However, interaction with mobile devices still relies on the tactile (input) and graphic (output) communication, which is established in the GUI. Through the analysis of the Tinder interface it is possible to explore which elements contribute to the application's interaction approach and to the establishment of an imbalanced gender dynamic (if any exists).

To understand how women feel using Tinder it was necessary to conduct a qualitative inquiry where the participants could describe their experience. Through this inquiry we could have a panoramic view of their experience. We posed open ended questions (see below) without a preconceived notion of a comprehensive catalogue of response categories they would elicit. However, inspection and reflection on the responses revealed patterns for individuals and across questions. The results enable quantification, which reveal, for example, the experience of offensive behavior related to gender bias by $70 \%$ of the participants. However, we also wanted to understand how the gender dynamics is manifested through the GUI and to point out which elements of the interface design contribute to the positive and negative side of their experiences.

To find out how users' impressions are related to the GUI we consider some theories related to design perception. According to the appraisal theory, people reveal their feelings through their appraisal of an event or product-in this case experience with the application. That is, their assessment of the experience reveals what they expect to feel while using the application, which enables the researchers to evaluate the Tinder experience through the female users' eyes. Appraisal of the achievement of a positive or negative experience depends on expectations being fulfilled or not [3]. Therefore, we focused on understanding women's expectations in order to analyze if the application meets their needs. In our study, the evaluation of the design relies on comparing the interaction process through the GUI with the users' expectations, impressions and feelings.

We also want to know how the interaction "affords" gender neutrality and pleasant experiences. How users perceive the interaction reveals not only their feelings but also the applications' affordances [4], which encompasses the design qualities [5]. In our study, we have identified a high frequency of offensive behaviors towards female users and have understood that this sort of behavior might be encouraged by the design of the application.

Gender bias can be intensified with Internet because people feel free to express their opinions with a certain level of anonymity. ${ }^{1}$ According to Wajcman [7], technology is an extension of a patriarchal structure that is shaped by men to exclude women. From her perspective, there is a tendency to reinforce gender hierarchies within the realm of technological developments. Considering this

\footnotetext{
${ }^{1}$ However, anonymity does not, in itself, assure negative social consequences; positive social consequences also derive from anonymous communications [6].
} 
scenario where women feel free to benefit from technological advances in a world still influenced by gender hierarchy, how should an application provide easy interactions and at the same time provide women empowerment, safety and respect?

\section{Methodology}

\subsection{Research Philosophy, Design and Methods}

Considering women's empowerment as a central subject matter of our study, we adopt a "transformative" [8] philosophy to guide the research, since the long-term goal (in establishing the impact of this and future research) is to draw attention to gender imbalance hidden in the shadow of technological developments that threatens women's confidence and do not provide an overall pleasant experience.

The research has a mixed methods approach incorporating both qualitative and quantitative data [8]. The approach would be considered qualitative regarding the subject and purpose of the study; however, we decided to integrate the quantitative approach to the analysis of the findings, which would help to establish facts about the distribution of perspectives on sensitive matters (female perception of an online dating application). The qualitative analysis made it possible to understand the gaps, problems and frustrations through female users' eyes. However, without the posterior qualitative analysis we would not be able to

judge if there is a problem regarding the experience and the presence of sexism in interactions. Because we first conducted the qualitative research and then use the quantitative approach to support the qualitative findings our research may be characterized as exploratory sequential mixed methods research [8].

The research method involves data generation [9] through the use of a structured interview with open-ended questions, text analysis of responses, the transformation of the interview information into statistical data and the analysis of the results. Although an unstructured interview might be recommended due the qualitative nature of the research questions [10] we considered the structured interview the best option to retain focus. The open-ended response approach enables elicitation of different points of view around each question [11]. We did not seek a broad analysis of user experience on Tinder, per se; rather, we wanted to investigate particular points regarding gender bias and gender dynamics. We conducted the interview via email, with a set of standard questions intended to elicit open-ended reply and to invite further interaction, to which participants could respond asynchronously, at their leisure. We reasoned that this would ensure the maximal amount of reflection and also reduce demand characteristics [12], along with other practical qualities of internet mediated research [13].

\subsection{Data Generation}

Our first step was to consider which would be the strategy to obtain the information we needed. Initially we considered a questionnaire. However, after reflection we decided that to create a questionnaire we would need to have already a deep 
understanding of the issues and anticipate all sorts of possible experiences to provide appropriate response categories. Thus, we considered a questionnaire as good tool for a later research stage, after collecting more information about all the possibilities of answers. However, the nature of the research is to understand how women feel about the application, and, considering this exploratory nature, we could not plan the later step without the findings of the first stage. Due to this, we decided to conduct a survey through structured interview. The design and administration of this interview (including recruitment methods and consent forms, etc.) were approved by the Research Ethics Committee of the School of Computer Science and Statistics of Trinity College Dublin. The recruitment was managed via online social media (OSM) and delivered by email.

\subsection{Recruitment}

Recruitment was conducted through Facebook. The first call for participants was posted in a Facebook closed group of Brazilian females living in Ireland. The group had 8,074 members at the time of recruitment (April, 2016). The call was posted only one time in this group. The invitation described the research topic and what would be involved in participating. Women interested in participating in the survey were asked to leave a comment or an inbox message with their email address. 111 women expressed interest. Another call was posted to friends in the personal Facebook page of one of the authors. From this call 14 women expressed interest. Some of these 125 original women invited another nine women to participate, and their emails were given with the permission of those nine. In total, 134 women expressed interest in participating in the research. The consent form approved by the ethics committee was sent to them all. However, not all responded with consent to the research terms and conditions and even fewer women have responded to the interview subsequent to providing informed consent. From the 134 total women that gave their emails only 40 were interviewed. Participation was voluntary, without remuneration.

This high drop-out rate could be attributed partly to the length of the information sheet and consent form that is necessary for proper ethical conduct. Attrition may also be partly attributed to the lack of immediacy in conducting interviews by email. Despite this, the interviewees are easily reached by email and it compensates for the drop-outs. We believe that in general the benefit of using the email to deliver the interviewees compensates for the losses.

\subsection{Participants}

The sample is qualified as a nonprobability convenience sample composed of forty Brazilian women who have used Tinder at least once to meet new people through the Internet. The convenience sample defines the unguided selection made through Facebook, since anyone could volunteer. All the volunteers had the chance to be interviewed. Although the findings cannot be extended to the whole population of women who use Tinder it reveals significant numbers that give a picture of the gender dynamics on Tinder. Age and education level were not 
taken into consideration in the selection of participants (we did not ask participants to provide such information about themselves), nor was gender preference; however, from the 40 women interviewed, one indicated looking for homosexual interaction. Nationality is a variable that may interact with outlook on the topics studied here, and our idea is to have a sample with mixed nationalities in future studies. However, due to the opportunity sampling (target population available and willing to take part in it) we used the population of Brazilian women.

\subsection{Structured interview}

The interview was planned by email since we believed women would feel more confident to communicate about their experiences through writing. Email administration of the interview afforded interviewees privacy to talk about the delicate themes involved. We also believed participants would have more time to think and remember their experiences before answering, since mediating interviews via email enables participants to respond at their leisure, with all of the opportunity for reflection that they might wish.

The interview guide was meant to be brief, to encourage people to answer completely. The interview was planned to be as neutral as possible. The questions were posed to elicit ample information. We reminded participants about the motivation of the research (to understand how women feel during their experience on Tinder). We then provided, in Brazilian Portuguese, the following questions at once to each participant, as a way of opening communication for further discussion. Below, we provide the questions in English translation, and indicate the motivations for each.

Question 1 - Why did you install the application? To analyze if Tinder is meeting expectations we need to know first what users expectations were. With this question we want to know their goals in using the application. However, this question focuses on a prior step, on the decision to instal it rather than its use, because we wanted participants to reflect about the moment before installation, too. One may easily install software without intending to use it, or with the intention of deferring use. One might wish to have something as a matter of principle, regardless of whether one uses it.

Question 2 - Did you have to ponder before installing it? Why? We wanted to know if there was any preconceived idea about the application, concerns or fears that could discourage installation. This question would yield indications of the level of influence that third parties would have on their choice of using the application (friends, partners, family, society in general).

Question 3 - When you started using it, what was your first impression? How did you feel about your first matches? The idea of this question is to know whether they like Tinder or not in the very first moments and, more generally, how they felt in their first experiences in recalling them.

Question 4 - How did you feel about the application's approach and the match-based interaction? This question aims to reveal what users think about the interaction and the interface. 
Question 5 - Which are the positive aspects of your experience? Tell me about some remarkable situations. We hoped participants would respond to this question with indication of what they saw as positive in their experience on Tinder. Those answers were compared to the expectations pointed out in the first question, in a later analysis.

Question 6 - Which are the negative aspects of your experience? Did anything unpleasant happen? With this question we wanted to learn categories of negative experiences on Tinder. Given our anticipation of sexist response among those negative experiences, we imagined that we would be able to identify the percentage of women who report having experienced a sexist behavior and to anticorrelate this with the feeling of being respected.

Question 7 - Did you feel respected as an individual and as a woman? Explain. This question was intended to elicit thoughts about feeling respected, not only by the application but also by the other users. Through this question we wanted to investigate whether Tinder creates a virtual space where sexist patterns of interaction are avoided or reproduced, and whether there is a clear relation between the response to this question and expression of positive and negative experiences. We wanted to know whether participants would "accept" some negative experiences related to gender bias and still feel respected, as gender dynamics is not only concerned with the male approach but also how women feel regarding to sexist behavior.

Question 8 - For how long have you been using/ have used the application? We hoped to measure expectation satisfaction with the partnerfinding application in relation to how long it would be retained.

Question 9 - If you have stopped using the application, what is the reason? We hoped responses to this question would reveal the reason to uninstall Tinder, the relation between expectations before the use and the fulfillment or not of their needs during the use, and the overall feeling of the whole experience.

Question 10 - Do you think the developer was a man or a woman? This question could reveal if the application was perceived as developed with consideration women's needs or not, considering the hypothesis that male dominance in technological development. We also wondered if participants would suggest that it was developed by a woman or mix-gendered team.

Question 11 - Do you see any difference between what man and woman expect from using the application? What are they looking for, in general? We ask about difference of general purpose to help us understand the relationship between their own purpose (expressed in response to the first question) and their expression of general purposes of use for each gender.

Question 12 - Do you have female friends that are using / have used the application? What did you heard from them? The idea of this question is to have an overall view of group of friends regarding the application. This provided an opportunity to relate their own use and experience of Tinder to the experience known in participants' circles of friends.

Question 13 - How could your experience be more pleasant? Users would point out the improvements they would like to see in the interface so 
they would have a better experience. The question was phrased in a way that would not rule out responses suggesting alternative contexts of use. However, in general, the responses attended to the interface rather than its context of use.

Question 14 - Apart from Tinder, have you used another relationship application? Which one do you prefer? We wanted to know how familiar the notion of online dating applications is to participants and how the Tinder experience rated in comparison to others.

\subsection{Data organization}

The empirical analysis and careful interpretation of the responses characterizes the qualitative approach to understand the female users' point of view and define the first stage of the research which is followed by a quantitative approach to handle data and interpret it statistically.

To understand how women feel during their experience on Tinder is a purely qualitative process. However, we needed to analyze the responses quantitatively so we could judge whether the overall experience is pleasant or not and whether the occurrence of gender bias was representative. We wanted to identify the percentage of women that gave similar answers to each question so we could determine whether Tinder was good for those users. We also wanted to know of patterns of response across questions. Due to the design of the study, in which we asked for detailed written responses, and for which the answers were broad and open-ended, we could not quantify the response with the raw data, but only in a categorization of the raw data.

The categorization process was to iteratively summarize the interviews responses, reducing paragraphs of reply to one or two sentences. The original fourteen questions were split into seventeen questions (since some of them had more than one constituent question) for analysis purposes. After that we repeatedly read the interviews to develop an exhaustive (but not mutually exclusive, in that a single person's response may include more than one category) categorization of answers. For example, for question 1, related to the purpose of use, found six response categories that we deem to encompass all 40 of the answers. The process was about narrowing down the options each time, grouping similar purposes and reducing them to the minimum number of options. In the process we tried to make sure that all the responses were well represented by the options, regardless the number of responses in each category. For example for question 1, one of the, interviewees indicated using Tinder to forget an ex-boyfriend. This response response differed from all the others'. To maintain rigor regarding this classification we could not infer that this respondant wanted to make friends or find a new relationship in order to reduce to five categories. We applied the category "other" to this response. Thus, we maintained six categories of response. We also reduced the six categories to a few words of description for each. For example from question 1, a sentence like "I've install the application because I was single and wanted to meet different people; I was looking for any kind of relationship, casual or not" was split into two categories "meet new people" and "find a relationship". After that, to handle the data conveniently in a statistical 
analysis package (R), we reduced that description to the word "relationship". Finally, we had to check if that group of answers would really encompass all the answers. It was necessary to return to the raw interview texts and verify that the options fit and certify that the information was not lost in the process of reducing paragraphs to words. After that, it was possible to organize the interview answers in a spreadsheet that was used to calculations: each participant in a separate row and the questions in separate columns.

After this transformation of the raw interview responses in a coded data set, we calculated the result of each column, that is, the sum of the equal replies for each question using R. As said before, the answers for each question were reduced to words and although the tabulations could have been done manually, we benefitted from using with $\mathrm{R}$ since it would be more reliable, besides providing the opportunity to find correlations through cross-tabulations.

For the questions in which a response fit more than one category, that question column was split into more columns, with each answer type in an individual cell for correct counting; otherwise, $\mathrm{R}$ would treat the compound entry as a new answer. Using this counting method, "find relationship and/or friends" is encoded with two columns, one for "find relationship" and another for "find friends". Creating additional columns enabled appropriate multiple response counting. After splitting all the possible response categories in columns, we could find the results for each question through a formula to make the sum of each category of answer in each question. For example, in question 5, regarding the positive experiences, there were women who pointed out four different "gains" and we could count the four columns in a unique result.

However, to make comparisons between two different questions it was necessary to use the original configuration of the responses (such as "find relationship and/or friends") to ensure that each response would represent a one woman. Otherwise, if we considered the total of responses (for "find friends" plus "find relationship" for example) we would have a higher number of women since each of them could have given more than one response for each question.

\section{Analysis and Findings}

As the purpose of this paper is to explain our mixed method research strategy, we do not highlight all of the results and findings. However, an overview is necessary to make clear the relation between the procedures and the results.

We calculated the percentage of each answer category and organized the data in tables for analysis. For example, we cross-tabulated responses to question 1 (purposes of use) and question 11 (the feeling about differences of purposes by gender). In this comparison we check if there is a correlation between the women's purposes and what they think regarding the difference about women and men's purposes in general. It shows that, from the women looking for relationship and/or friends but not for casual sex (31 women), 20 believe that men are using the application for different a purpose than theirs. Thus, $2 / 3$ of the women who are only looking for relationship and/or friendship believe that men are not. This 
result suggest that Tinder can have a "sexual" approach that does not fit in their needs and expectations (regardless their gains during their experience) since 39 women from the original sample were looking for heterosexual interaction.

The results showed that $52.6 \%$ of the female users had a negative first impression and the superficiality of the interface was the most rated problem. The superficiality was related to the ease of Internet that is emphasized by Tinder with a very simple interaction based on choice restricted to between two options: "yes" and "no". This approach which can be "simple and easy" for some users, highlights the concept of liquidity of relationships [14] mediated via the Internet. The fragility of emotional bonds is related to the superficiality of relationships, and superficiality in turn can directly affect feelings of respect, politeness and social dynamics. This idea is magnified in online dating applications due to the perception of Internet as a "free- zone" (virtual social context), where people feel more free to say what they would not dare to say in other circumstances (and real social contexts). Harassment, for example, brings to light the "predatorprey" dynamic in which women are considered "vulnerable". This dynamic is created during childhood when "little innocent girls" are often "in danger" and should be protected from sexual abuse [15]. Nonetheless, the distorted idea of "vulnerability" has implication in adult life, and it is continued when women are taken as an object of uncontrolled male sexuality. For example, in our research $70 \%$ of the participants reported offensive behavior regarding gender dynamics and pointed it out as the worst aspect of their experience.

When asked about feeling respected, only 9 women from 28 respondants expressed feeling mostly or fully respected, the other 21 expressed feeling "more or less", "mostly not" or "not" respected. This results shows that the majority does not feel "mostly" or "fully" respected.

Regarding their overall feeling after use it became clear that Tinder does not meet their needs, in general, although it is efficient on delivering "fragile relationships". From 35 women who answered the question, 20 have uninstalled the application due to a negative experience (frustration, loss of interest and bad feelings after repressed by other users); two due a mix of negative experience and the start of a relationship; and 13 have quit the application only because they started a relationship. The $57.1 \%$ who removed the application exclusively because of a negative experience constitutes a majority of the interviewees.

In relation to the first objective of the research (understanding how women feel using Tinder), it was possible to identify that Tinder does not provide an overall pleasant experience to women, since all these results point to the feeling of frustration: the results of the first impressions about the application, the analysis of negative experiences, the feeling of respect, the comparison between user's motivations and the perception of difference of motivations between men and women, and the reason to uninstall Tinder. Concerning the second objective of the research (identifying the existence of gender bias), the results of the negative side of their experience (related above), the feeling that it was designed by a man by $85.3 \%$ of the respondants, the feeling of not being mostly or fully respected by the majority of the respondants, and the $35.90 \%$ of respondants 
that was concerned about installing Tinder due to its sexual approach and bad reputation all point to gender bias on Tinder. We also have noted patterns of response suggesting that the Tinder GUI and interaction encourage negative experiences (cf. the third objective of the research): the GUI which encourages the discarding of people and reinforces superficiality; the gesture set is merely binary, leaving no way to express nuance; and so on. However, because the focus here is to illustrate our method of forming qualitative assessments, informed by quantitative analysis of qualitative data, we will limit the descriptions of the findings and analysis to the paragraphs above.

\section{Contributions, Limits and Challenges}

The research reported here is exploratory as an initial study of gender and online dating application that requires further development to ensure quality and rigor. However, it is the first of its kind that we know of. Our findings support the hypothesis that Tinder harbours sexist behaviors, though it is possible that conclusion exemplifies confirmation bias. The research requires a following work to investigate the gender bias more deeply and with a vastly larger number of participants. As the study includes only 40 women it is not enough for a generalization of the experience. We have not quantified the dimensions along which users did not experience gender oriented challenges at all, nor the dimensions the contributed gender-oriented support. Further studies would require carrying out other experiments as focus groups, user tests, user diaries and empirical evaluation to deepen the understanding of the gender dynamics through the use of online dating applications.

The results of this research bring out some reflections about the dynamic established by an online dating application that would enable future improvement of the system. However, this first investigation gives an overall idea of the problems that affects the women's experience. It addresses design implications to thwart sexist behavior and to improve women's experience. Besides the problems revealed by the research, Tinder has proven to be effective in delivering romantic relationships and friendship and to be a powerful tool to connect people. Some boundaries are mitigated through the application, enabling serendipitous engagements that address a positive quality to the use of technology in the realm of relationship. The results of this first survey indicates that the topic (gender bias) is important and it should be better developed and explored to enhance the women empowerment within technological field and its products. The graphical user interface guides the interaction and can be a source for balancing the gender differences in everyday interactions.

\section{Acknowledgements}

The researchers have no conflicts of interest with Tinder nor any comparable platform. The first author wishes to acknowledge the Brazilian government's CAPES program. This research is supported by Science Foundation Ireland (SFI) through the 
CNGL Programme (Grant 12/CE/I2267 and 13/RC/2106) in the ADAPT Centre (www.adaptcentre.ie).

\section{References}

1. Petersen, M.G., Iversen, O.S., Krogh, P.G., Ludvigsen, M.: Aesthetic interaction: a pragmatist's aesthetics of interactive systems. In: 5th conference on Designing interactive systems: processes, practices, methods, and techniques (DIS '04) (2004)

2. Te'eni, D., Carey, J., Zhang, P.: Human-Computer Interation: Developing Effective Organizational Information Systems. John Wiley \& Sons (2007)

3. Fridja, N.H.: The Laws of Emotion. Lawrence Erlbaum, Mahwah, NJ (2007)

4. Gibson, J.J.: The ecological approach to visual perception. Houghton Mifflin, Boston, MA (1979)

5. Norman, D.: The psychology of everyday things. Basic Books, New York, NY (1988)

6. Vogel, C.: Anonymous freespeech. In: K. Ahmad, C. Vogel (eds.) DIMPLE:DIsaster Management and Principled Large-scale information Extraction for and post emergency Logistics, pp. 26-31. The 9th edition of the Language Resources and Evaluation Conference (2014). Reykjavik, Iceland, May 26-31, 2014

7. Wajcman, J.: Technofeminism. Polity Press, Cambridge, UK (2004)

8. Creswell, J.W.: Research Design: Qualitative, Quantitative, and Mixed Methods Approach. SAGE (2014)

9. Birks, M., Mills, J.: Grounded Theory: A practical Guide. SAGE (2015)

10. Corbin, J., Strauss, A.: Basics of Qualitative Research: Techniques and Procedures for developing Grounded Theory. SAGE (2008)

11. Patton, M.Q.: Qualitative Research and Evaluation Methods. SAGE (2002)

12. Rosenthal, R., Rosnow, R.L.: Artifacts in Behavioral Research. Reissue of Artifact in Behavioral Research, Experimenter Effects in Behavioral Research and The Volunteer Subject. Oxford University Press (2009)

13. Hewson, C., Vogel, C., Laurent, D.: Internet Research Methods, 2nd Ed. London: SAGE (2016)

14. Bauman, Z.: Liquid Love: on the frailty of human bonds. Polity Press, Cambridge, UK (2003)

15. Walkerdine, V.: Daddy's Girl: Young Girls and Popular Culture. MacMillan Press LTD, London, UK (1997) 